\title{
Coherent $x$-ray transition and diffraction radiation of microbunched beams
}

\author{
K. A. Ispirian ${ }^{1}$ and M. Ispiryan ${ }^{2, *}$ \\ ${ }^{1}$ Alikhanian National Laboratory (Yerevan Physics Institute), Brothers Alikhanian 2, Yerevan, 0036, Armenia \\ ${ }^{2}$ University of Pennsylvania, Philadelphia, Pennsylvania 19104, USA
}

(Received 27 September 2011; published 5 February 2013)

\begin{abstract}
Theoretical and numerical results on angular and spectral distributions and total number of photons of several types of coherent radiation produced by microbunched beams passing through radiators are presented: coherent $x$-ray bremsstrahlung, $x$-ray transition, resonance transition, and diffraction radiations. The possibility of observation and application of these new types of radiation for the study of parameters of electron beam microbunching, which is important for the effectiveness of $\mathrm{x}$-ray free electron lasers, is discussed.
\end{abstract}

When a charged particle passes through or close to the interface between two media with different dielectric constants, then, due to the transformation of the electromagnetic field of the charged particle, transition and related diffraction radiations (TR and DR) are produced with broad spectrum [1-3]. Bunches of $N_{b} \gg 1$ particles will emit coherent TR with intensity proportional to $N_{b}^{2}$ at wavelengths larger or equal to the bunch length (for review and derivations, see [4]). Because of interference, a periodic train of bunches of electrons microbunched with a frequency $\omega_{r}$ will emit intense TR photons, again proportional to $N_{b}^{2}$, in a very narrow spectral range around $\omega_{r}$ in soft [5] and x-ray regions [6].

After a proposal and subsequently conducted experiments $[7,8]$, the optical transition radiation has found wide application in high energy particle beam diagnostics. Recently, following the proposal [5], the coherent optical and softer TR produced by microbunched particles has found application for the study of particle grouping or microbunching $(\mathrm{MB})$ in existing soft photon free electron lasers (FELs) [9,10]. After the proposals [11] and experiments $[1,2]$, the $\mathrm{x}$-ray transition radiation (XTR) has found wide application in identification of single high energy particles. Recently, it has been shown [6] that coherent XTR (CXTR) can also be used for the study of MB at existing (LCLS, SLAC) [12] and future free electron lasers (see Table 1 of [13]). However, the outcomes of corresponding experiments are not known yet, though there exist a proposal [14] and even results of a preparatory work [15].

The method of calculation of the angular-spectral distributions of the coherent radiation $d^{2} N_{\mathrm{CXR}} / d \omega d \theta$ of

\footnotetext{
*Corresponding author.

misp@mail.med.upenn.edu
}

Published by the American Physical Society under the terms of the Creative Commons Attribution 3.0 License. Further distribution of this work must maintain attribution to the author(s) and the published article's title, journal citation, and DOI. interest with the help of known incoherent distributions $d^{2} N_{\mathrm{XR}} / d \omega d \theta$ consists in substitution of $d^{2} N_{\mathrm{XR}} / d \omega d \theta$ into the formula from [4]:

$$
\frac{d^{2} N_{\mathrm{CXR}}}{d \omega d \theta} \approx N_{b}^{2} F(\omega) \frac{d^{2} N_{\mathrm{XR}}}{d \omega d \theta}
$$

where, after [5,6],

$$
\begin{aligned}
F_{F F}(\omega, \theta)= & \exp \left[-\left(k \sigma_{r} \sin \theta\right)^{2}\right]\left\{\exp \left(\frac{-\omega^{2} \sigma_{z}^{2}}{2 v^{2}}\right)\right. \\
& +b_{1} \exp \left[-\left(\frac{\omega}{v}-\frac{\omega_{r}}{c}\right)^{2} \frac{\sigma_{z}^{2}}{2}\right] \\
& \left.+b_{1} \exp \left[-\left(\frac{\omega}{v}+\frac{\omega_{r}}{c}\right)^{2} \frac{\sigma_{z}^{2}}{2}\right]\right\}^{2}
\end{aligned}
$$

is the form factor of the Gaussian electron bunch with radial $\sigma_{r}$ and longitudinal $\sigma_{z}$ rms deviations; $b_{1}$ and $\omega_{r}$ are the MB parameter and frequency, respectively; $v$ is the electron velocity.

In brief, the physics of the production of various types of coherent radiation by these microbunches is the following. In the beginning, let us assume that $N_{B}=M n_{b}$ is the number of electrons in a pulse or macrobunch consisting of $M$ very narrow microbunches each containing $n_{b}$ electrons with distance between each other just equal to $\lambda_{r}$. It is well known that in this case all the $n_{b}$ electrons of the first microbunch oscillate synchronously and radiate photons coherently. The number of photons is proportional to $n_{b}^{2}$ at wavelengths larger or equal to the width $\Delta L_{\text {micro }}$ of the microbunches if $\Delta L_{\text {micro }} \ll \lambda_{r}$. The following microbunches also radiate independently and in the same way. However, since the microbunches are periodical with distance $\lambda_{r}$ between them, then, due to interference, the summary radiation of the macrobunch will be enhanced coherently only at $\lambda=\lambda_{r}$ and incoherently at other frequencies. The number of the enhanced coherent radiation photons from all the microbunches is not equal to the sum of the photon numbers from each of the microbunches; instead of being $M n_{b}^{2}$ times greater than the intensity from 
a single electron, it is $M^{2} n_{b}^{2}=N_{b}^{2}$ times greater than the number from single electrons. However, if the microbunches are not very narrow, for instance, $\Delta L_{\text {micro }}<\lambda_{r}$, then the coherent radiation from each microbunch will be added partially coherently, and the total number of the radiated photons from the macrobunch will be proportional to $K M^{2} n_{b}^{2}=K N_{b}^{2}$, where the coefficient $K$ is less than 1 .

Considering only the frequency region around $\omega_{r}$ where one expects strong enhancement, the first and the third terms in the square brackets of (2) can be neglected, while the important second term predetermines the widths of the spectra. To derive the angular distribution (AD) $d N_{\mathrm{CXR}} / d \theta$, it is necessary to integrate $d^{2} N_{\mathrm{CXR}} / d \omega d \theta$ over $\omega$, pulling out the weakly varying parts from under the integral and replacing $\omega$ by $\omega_{r}$. The remaining part of the integral yields $v \sqrt{\pi} / 2 \sigma_{z}$. To derive the spectral distributions (SD) $d N_{\mathrm{CXR}} / d \omega$ and the total number of coherent photons $N_{\text {CXR }}$, it is necessary to integrate $d^{2} N_{\text {CXR }} / d \omega d \theta$ over $\theta$ and $\omega$ analytically or numerically, respectively.

This work is devoted to the first theoretical study of CXBR (coherent X-ray bremsstrahlung), CXRTR (coherent $\mathrm{X}$-ray resonance transition radiation), and CXDR (coherent $\mathrm{x}$-ray diffraction radiation), as well as to the confirmation of the results [6] on CXTR with the purpose to find new methods of measurement of $b_{1}$ of microbunched beams. Methods of diagnostics of not-microbunched particle beams will not be considered (see, for instance, [16] and references therein).

Let us begin with the coherent bremsstrahlung, CXBR, which is a background during the measurements. Since the MB electrons have $\sim \mathrm{GeV}$ energies, while the CXBR consists of (1-10) $\mathrm{keV}$ photons, it is necessary to use relativistic formulas for bremsstrahlung, taking into account the density or medium polarization effect [2]. Using a technique from [17], we factor $d^{2} N_{\mathrm{BR}} / d \omega d \theta$ into the product of angular and frequency parts. The first is the classical cross section of the $\mathrm{AD}$ of the Rutherford elastic scattering. The second is the probability of photon radiation, for which, despite [17], we use the QED Born approximation formula taking into account the density effect [2] instead of the formula without the polarization effect. With these, we have

$$
\frac{d^{2} N_{\mathrm{BR}}}{d \omega d \theta}=\frac{A_{\mathrm{BR}}}{A_{\text {norm }}} F(y) F 1(\omega),
$$

where $y=\gamma \theta$ [the Lorentz factor $\gamma=E / m c^{2}=1 /$ $\left(1-\beta^{2}\right)^{1 / 2}$ ], and, according to [2,17],

$$
\begin{aligned}
F(y) & =\gamma \frac{y\left(1+y^{4}\right)}{\left(1+y^{2}\right)^{4}} \\
F 1(\omega) & =\frac{1}{\omega}\left[\left(\frac{\hbar \omega}{E}\right)^{2}+\frac{4}{3}\left(1-\frac{\hbar \omega}{E}\right)\right] \frac{1}{1+\gamma^{2}\left(\omega_{P} / \omega\right)^{2}} .
\end{aligned}
$$

$A_{\mathrm{BR}}=12 N_{0} \bar{\sigma} L_{\mathrm{rad}} L ; N_{0}$ is the number of atoms in unit volume of the radiator with thickness $L$ (it is reasonable to take $L \approx 3 L_{\text {abs }}$ where $L_{\text {abs }}$ is the absorption length of resonance photons); $\bar{\sigma}=Z^{2} \alpha r_{e}^{2}=Z^{2} \times 5.8 \times 10^{-28} \mathrm{~cm}^{2} ; Z$ is the atomic number; $A_{\text {norm }}=1 / 3$ is a normalization factor; $\omega_{p}$ is the plasma frequency. Following (6), it is helpful to introduce two dimensionless parameters $q_{\text {dif }}=\omega \sigma_{r} / v$ and $\tau=\gamma / q_{\text {dif }}$.

Using the above described methods for $\tau \ll 1$, a condition which almost always takes place, one obtains

$$
\begin{aligned}
& \frac{d^{2} N_{\mathrm{CXBR}}}{d \omega d \theta}= 3 N_{B}^{2} b_{1}^{2} A_{\mathrm{BR}} F(y) F 1(\omega) \exp \left[-\left(\frac{y}{\tau}\right)^{2}\right] \\
& \times \exp \left[-\left(\frac{\omega}{\nu}-k_{r}\right)^{2} \sigma_{z}^{2}\right] \\
& \frac{d N_{\mathrm{CXBR}}}{d y}=N_{B}^{2} b_{1}^{2} C \exp \left(-y^{2} / \tau^{2}\right) y \frac{1+y^{4}}{\left(1+y^{2}\right)^{4}} \\
& \frac{d N_{\mathrm{CXBR}}}{d \omega}=3 N_{B}^{2} b_{1}^{2} A_{\mathrm{BR}} C \tau^{2} F 1(\omega) \exp \left[-\left(\omega / \nu-k_{r}\right)^{2} \sigma_{z}^{2}\right]
\end{aligned}
$$

$$
N_{\mathrm{CXBR}}=N_{B}^{2} b_{1}^{2} C \tau^{2} / 2,
$$

where $C=3 A_{B r} v \sqrt{\pi} F 1_{\omega}\left(\omega_{r}\right) / \sigma_{z}$. The numerical results on usage of (5)-(8) will be discussed below.

In the same way, using the well-known formula for XTR for a single interface [3], one obtains the following densities and number of photons for CXTR produced by a microbunched beam on single interface when $\tau \ll 1$ (see also [6]):

$$
\begin{aligned}
\frac{d^{2} N_{\mathrm{CXTR}}}{d \omega d \theta}= & \frac{2 \alpha \alpha_{b}^{2} b_{1}^{2}}{\pi} \frac{\omega_{P}^{4}}{\omega^{5}} \frac{\theta^{3}}{\left(\gamma^{-2}+\theta^{2}\right)^{2}\left(\gamma^{-2}+\theta^{2}+\omega_{P}^{2} / \omega^{2}\right)^{2}} \\
& \times \exp \left[-\left(q_{\mathrm{dif}} \theta\right)^{2}\right] \exp \left[-\left(\frac{\omega}{v}-\frac{\omega_{r}}{c}\right)^{2} \sigma_{z}^{2}\right] \\
\frac{d N_{\mathrm{CXTR}}}{d \theta}= & \frac{2 \alpha \alpha_{B}^{2} v \omega_{p}^{4}}{\sqrt{\pi} \sigma_{z} \omega_{r}^{5}} \frac{\theta^{3}}{\left(\gamma^{-2}+\theta^{2}\right)^{2}\left(\gamma^{-2}+\theta^{2}+\omega_{P}^{2} / \omega^{2}\right)^{2}} \\
& \times \exp \left[-\left(q_{\mathrm{dif}} \theta\right)^{2}\right] \\
\frac{d N_{\mathrm{CXTR}}}{d \omega}= & \frac{\alpha N_{B}{ }^{2} b_{1}^{2} \tau^{4}}{\pi \omega} \exp \left[-\left(\frac{\omega}{v}-k_{r}\right)^{2} \sigma_{z}^{2}\right] \\
N_{\mathrm{CXTR}} \approx & \frac{\alpha N_{B}{ }^{2} b_{1}{ }^{2} v}{\sqrt{\pi} \sigma_{z} \omega_{r}} \frac{\tau^{4}}{\left(1+\omega_{\gamma}^{2}\right)^{2}} .
\end{aligned}
$$

Similarly for CXRTR, when the microbunched beam passes through a stack of $M$ pairs of two plates with thicknesses $l_{1,2}$, linear absorption coefficients $\mu_{1,2}$ and formation lengths $Z_{1,2}$ of the XTR, one obtains

$$
\begin{aligned}
\frac{d^{2} N_{\mathrm{CXRTR}}}{d \omega d \theta}= & N_{B}^{2} b_{1}^{2} F_{1}(\omega, \theta) F_{2}(\omega, \theta) F_{3}(\omega, \theta) \\
& \times \exp \left[-\left(\omega \omega_{r} \theta / v\right)^{2}\right] \exp \left[-\left(\omega / v-\omega_{r} / v\right)^{2} \sigma_{z}^{2}\right],
\end{aligned}
$$




$$
\begin{aligned}
\frac{d N_{\mathrm{CXRTR}}}{d \theta}= & N_{B}^{2} b_{1}^{2} \frac{v \sqrt{\pi}}{2 \sigma_{z}} F_{1}\left(\omega_{r}, \theta\right) F_{2}\left(\omega_{r}, \theta\right) F_{3}\left(\omega_{r}, \theta\right) \\
& \times \exp \left[-\left(\omega_{r} \sigma_{r} \theta / v\right)^{2}\right],
\end{aligned}
$$

where $[3,18]$

$$
\begin{gathered}
F_{1}(\omega, \theta)=\alpha \omega \theta^{3}\left(Z_{1}-Z_{2}\right)^{2} / 8 \pi \mathrm{c}^{2}, \\
F_{2}(\omega, \theta)=1+\exp \left(-\mu_{1} l_{1}\right)-2 \exp \left(-\mu_{1} l_{1} / 2\right) \cos \left(l_{1} / Z_{1}\right),
\end{gathered}
$$

$$
F_{3}(\omega, \theta)=\frac{1+\exp (-M \sigma)-2 \exp (-M \sigma / 2) \cos (2 M X)}{1+\exp (-\sigma)-2 \exp (-\sigma / 2) \cos (2 X)}
$$

$$
\begin{gathered}
\sigma=\mu_{1} l_{1}+\mu_{2} l_{2}, \\
X=l_{1} / Z_{1}+l_{2} / Z_{2}, \\
Z_{1,2} \approx \frac{4 \beta \mathrm{c}}{\omega\left[\gamma^{-2}+\left(\omega_{p 1,2} / \omega\right)^{2}+\theta^{2}\right]} .
\end{gathered}
$$

Unfortunately $d N_{\text {CXRTR }} / d \omega$ and $N_{\text {CXRTR }}$ can only be found by numerical integration. Some results will be given below.

Finally, let us consider CXDR after making some preliminary remarks. The first theoretical works on DR of high energy particles were published long ago (see [2]). It is interesting to note that experimental works on DR produced by high energy particles have begun after work [19], in which coherent (the wavelengths are larger than the beam sizes, and the intensity is $\sim N_{b}^{2}$ ), long wave DR has been observed, in the millimeter wavelength range. Among recent experimental results on DR it is worthwhile to mention the work [20], carried out with nonrelativistic electron beam with $\sim 20 \mathrm{~nm}$ cross section passing through a $\sim 100 \mathrm{~nm}$ hole in a multilayer. At present, optical and softer DR is used for the noninvasive and noninterceptive measurement of the beam transversal phase volume and of the longitudinal sizes of bunches.

Let us also note that, in contrast to TR, before the works $[21,22]$, the DR has not been studied in the x-ray region. In [21], on the basis of some considerations given in [2], it has been shown that the XDR can be produced only for ideal, nonexisting beams. However, the authors of [21] have shown theoretically that XDR-a phenomenon close to XTR - can be produced by relativistic particles. However, while the XTR's intensity decreases sharply after the critical frequency $\omega_{\text {crit }}^{\mathrm{XTR}} \approx \gamma \omega_{P}$ proportional to $\omega^{-4}$, the critical frequency of XDR is equal to $\omega_{\text {crit }}^{\mathrm{XDR}} \approx c \gamma / H$, where $H$ is the impact parameter, by definition equal to the minimal distance between the particle's trajectory and radiator's edge. According to [21,22], the intensity of XDR after $\omega_{\text {crit }}^{\mathrm{XDR}}$ decreases proportional to $\exp \left(-2 \omega / \omega_{\text {crit }}^{\mathrm{XDR}}\right)$. Therefore, the XDR has very low intensity for $H$ of the order of a few micrometers. Nevertheless, one of the purposes of this work is to show that, as in the case of soft CDR [19], apparently the x-ray DR can be observed as CXDR of MB beams when the intensity is again proportional to $N_{b}^{2}$, and the spectrum is very narrow. Indeed, using the above methods and formulas from [21,22] for incoherent XDR for the case when the well focused microbunched beam passes perpendicular to the strip radiator with thickness $a$ and width $b$ (see inset of Fig. 3), one obtains for CXDR

$$
\begin{aligned}
\frac{d^{2} N_{\mathrm{CXDR}}}{d \omega d \theta}= & N_{b}^{2} b_{1}^{2} \frac{\alpha \omega_{P}^{4} \gamma^{4}}{\pi} \frac{y}{\omega^{5}} \\
& \times \frac{\sin ^{2}\left\{\frac{a}{4}\left(\frac{\omega}{c \gamma^{2}}\right)\left[1+y^{2}+\left(\gamma \omega_{P} / \omega\right)^{2}\right]\right\}}{\left(1+y^{2}\right)\left[1+y^{2}+\left(\gamma \omega_{P} / \omega\right)^{2}\right]^{2}} F(y, \omega) \\
& \times \exp \left[-\left(\frac{\omega}{c \gamma} \sigma_{r} y\right)^{2}\right] \exp \left[-\left(\frac{\omega}{v}-\frac{\omega_{r}}{c}\right)^{2}\left(\frac{\sigma_{z}}{c}\right)^{2}\right] .
\end{aligned}
$$

Here

$F(y, \omega)=\int_{0}^{2 \pi} \frac{1+2 y^{2} \cos ^{2} \phi}{1+y^{2} \cos ^{2} \phi} \exp \left[-\frac{2 H \omega}{\gamma c} \sqrt{1+y^{2} \cos ^{2} \phi}\right] d \phi$

is an integral over the azimuthal angle $\varphi$, which, unfortunately, can be calculated only numerically. From work [21], it follows that (21) and (22) do not depend on $b$ for DR radiators thicker than $\sim 1 \mathrm{~mm}$.

The sine square factor in (21) oscillates rapidly; hence, for

$$
\omega \approx \omega_{r} \ll \gamma \omega_{P}
$$

one can take its mean value $1 / 2$ if $a H \pi^{2} \gg \gamma \lambda_{P}$, where $\lambda_{P}=2 \pi \mathrm{c} / \omega_{P}$ is the plasmon wavelength of the radiator.

Again, $d N_{\mathrm{CXDR}} / d \theta, d N_{\mathrm{CXDR}} / d \omega$, and $N_{\mathrm{CXDR}}$ can be calculated only numerically.

The numerical calculations below are made for the following parameters of LCLS [12]: $E=13.6 \mathrm{GeV}$, $\hbar \omega_{r}=8.3 \mathrm{keV}, \sigma_{z}=9 \times 10^{-4} \mathrm{~cm}$ (FWHM length equal to $2.1 \times 10^{-3} \mathrm{~cm}$ or $70 \mathrm{fs}$ ), $\sigma_{r}=6.12 \times 10^{-4} \mathrm{~cm}$ (this value can be obtained taking the given $\gamma \varepsilon_{r}=0.5 \mu \mathrm{m}$ and $\left.\sigma_{r^{\prime}}=1 \mu \mathrm{rad}\right), N_{B}=1.56 \times 10^{9}$, and $b_{1}=1$. The radiators for CXTR, CXBR, and CXDR are titanium foils with $\hbar \omega_{P}=4.16 \times 10^{-2} \mathrm{keV}, \quad a=0.0035 \mathrm{~cm}, \quad b=2 \mathrm{~mm}$,

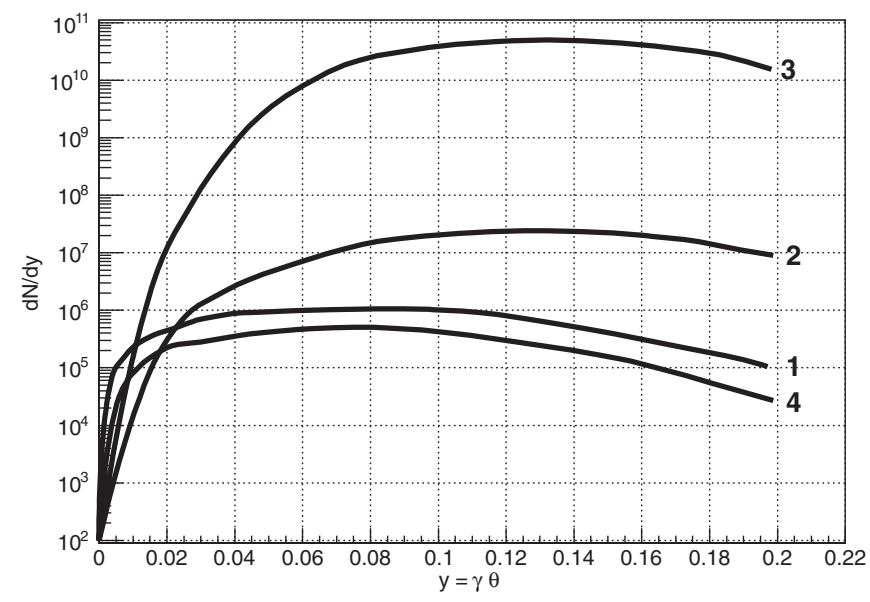

FIG. 1. The angular distributions of CXBR, CXTR, CXRTR, and CXDR (curves 1, 2, 3, and 4, respectively). 


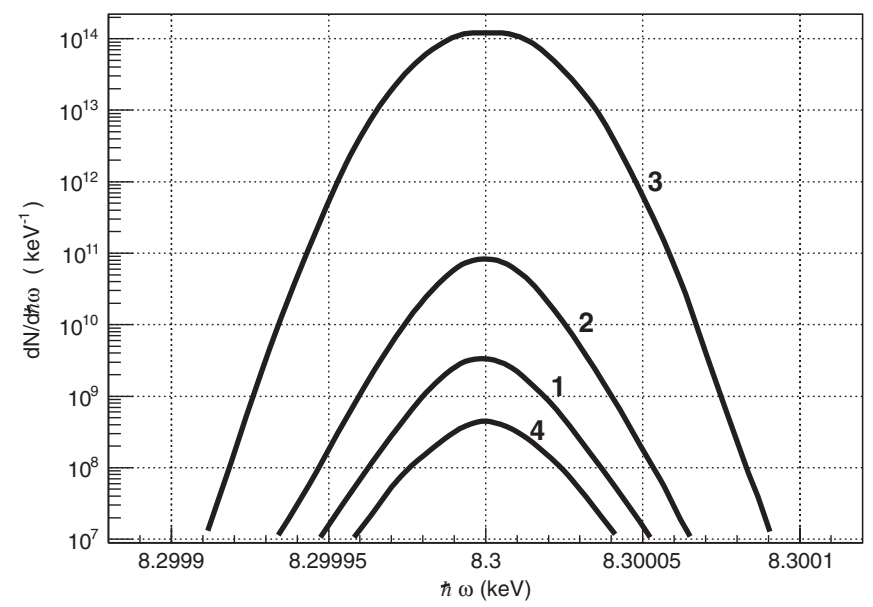

FIG. 2. Spectral distributions of CXBR, CXTR, CXRTR, and CXDR (curves 1, 2, 3, and 4, respectively).

and for the value $H=3 \mu \mathrm{m}$, while for CXRTR a stack of $M=270$ beryllium foils with $l_{1}=0.005 \mathrm{~cm}$ and $l_{2}=$ $0.02 \mathrm{~cm}$ is used.

Figure 1 shows the ADs of CXBR, CXTR, CXRTR, and CXDR produced by one macropulse and calculated with the help of (6), (10), and (14) and numerical integration of (21). It is seen that the angles corresponding to maximal intensity of various types of radiation $(y=0.1-0.15$ or $\theta \sim(0.1-0.15) / \gamma)$ are much smaller than those for incoherent radiation $(y \sim 1$ or $\theta \sim 1 / \gamma)$ and differ from each other. This fact, as well as the fact that these angles are greater than XFEL (FEL producing $\mathrm{X}$-ray beams) radiation angles, gives us a hope that these new types of radiation can be observed.

Figure 2 shows the SD of CXBR, CXTR, CXRTR, and CXDR calculated with the help of (7) and (11) and numerical integration of formulas (13) and (21).

From Figs. 1 and 2 it follows that during the measurement of the CXTR and especially CXRTR, the CXBR background is always small, while during the measurements of CXDR the CXBR background is absent since the beam bypasses the DR radiator.

The total numbers of the emitted photons per one bunch calculated with the help of (8) and (12) and integration of (13) and (21) are equal to $N_{\mathrm{CXBR}}=2.8 \times 10^{5}, N_{\mathrm{CXTR}}=$ $6.7 \times 10^{6}, N_{\text {CXRTR }}=2 \times 10^{9}$, and $N_{\text {CXDR }}=3.4 \times 10^{4}$.

Schemes of possible experimental arrangements designed for detection of these radiations (a) without (as in [14]) and (b) with separation of the microbunched electron beam from the accompanying SASE radiation are shown in Fig. 3. In the case (a), the presence of the shown magnetic system conserving $\mathrm{MB}$ of the deflecting magnet $M$, the dump and multilayer mirror ML1 are not necessary. Part of the radiation produced in the radiator and emitted around $\theta_{\max }$ is detected with the help of x-ray detectors $D$ of a Bragg spectrometer with annular conical crystal $C$ or an assembly of flat crystals. The difference from the scheme of [14] is the crystals $C$. Varying the distance $L$ between the radiator $R$ and $C$ or the distance $r$ between the arrangement axis and $C$, one can choose $\Delta \theta_{\text {opt }}$, the optimal angular interval for the type of radiation under investigation (see Fig. 1). However, in this case the contribution of the stimulated radiations of the electron beam under the influence of

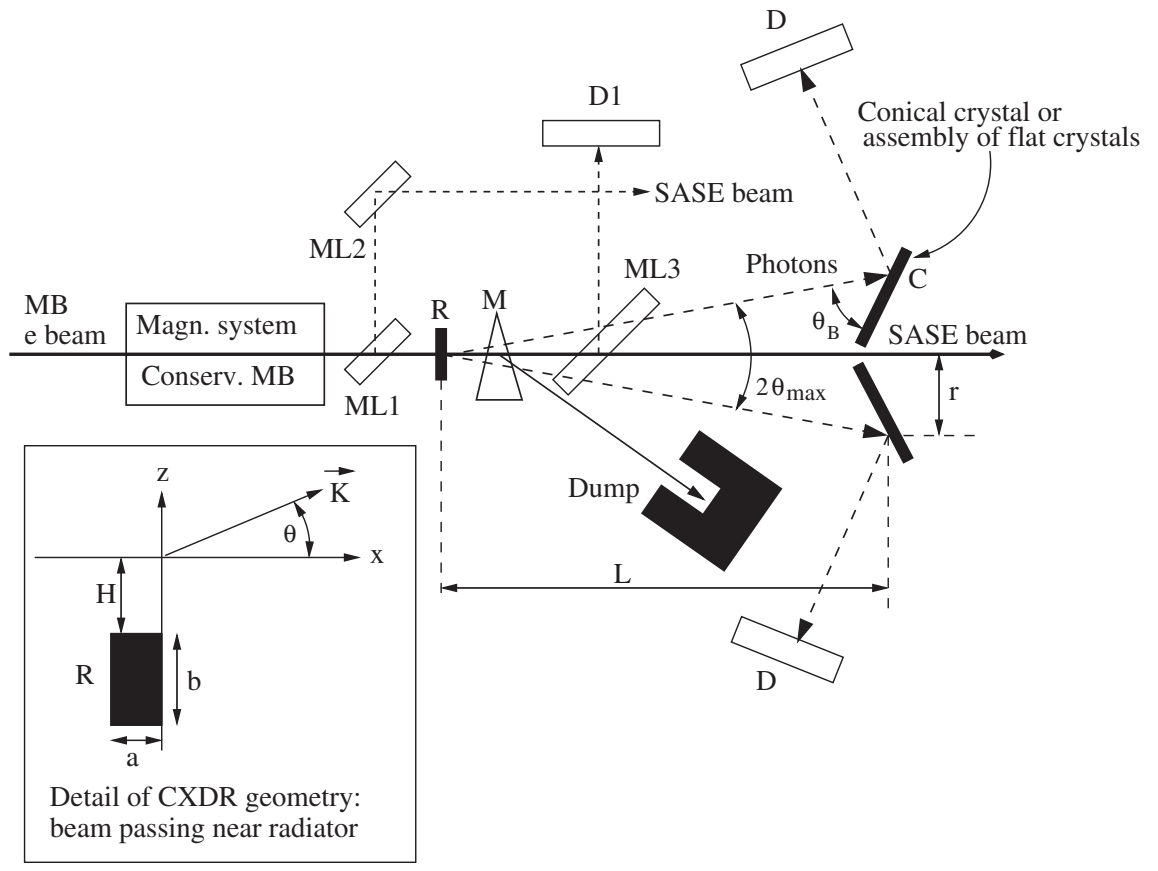

FIG. 3. Possible arrangements for study of CXBR, CXTR, and CXRTR at LCLS. The inset shows details of CXDR geometry. 
accompanying intense XFEL radiation remains unknown until the end of our ongoing work on calculations of this stimulated radiation, similar to radiation considered in [23].

In the case (b), the microbunched electron beam after the long undulator of the XFEL is separated from the XFEL SASE radiation with the help of a magnetic system conserving MB [24-26], or the SASE beam is deflected with the help of multilayer x-ray mirrors, ML1 and ML2. The pure electron beam after passing the radiator $R$ is deflected by the magnet $M$ and is sent to the dump. The coherent radiation photons produced in the radiator $R$ are deflected by multilayer mirror ML3 and detected with the help of $\mathrm{x}$-ray detector $D 1$. If the intensity of the produced coherent radiation photons appears to be high, then, in order to escape a pileup effect, the mirror ML2 can be replaced by a thin Compton scattering target, and the scattered photons with less intensity can be detected with $D 1$.

We now come to the following brief conclusions. (1) Though many factors, such as multiple scattering, instabilities, $\Delta \omega_{r}$, etc., were not taken into account, nevertheless, all these types of radiation, especially CXTR and CXRTR, can serve for the study of MB in XFELS. (2) The CXBR background is small while measuring CXTR, CXRTR, and CXDR. We keep in mind that in the case of CXDR the electron beam does not pass through the radiator. (3) All the calculations carried out above have been performed assuming $b_{1}=1$. Therefore, even in the case of small value of $b_{1}=10^{-2}$, the statistics allows us to measure $N_{\text {CXTR }}$ with its value being $N_{\mathrm{CXTR}}=6.7 \times 10^{2}$ in the presence of the background CXBR with $N_{\mathrm{CXBR}}=2.8 \times 10^{1}$. (4) For the first time it has been shown that the still-not-observed $x$-ray DR can be observed and studied due to the large enhancement factor $f=N_{b}^{2} b_{1}^{2}$.

\section{ACKNOWLEDGMENTS}

The authors thank M. A. Aginian, X. Artru, and L. A. Gevorgian for discussions and help.

[1] V. L. Ginzburg and I. M. Frank, Zh. Eksp. Teor. Fiz. 16, 15 (1946).

[2] M. L. Ter-Mikaelian, The Influence of the Medium on High Energy Processes at High Energies (Publishing House of Academy of Science of Armenia, Yerevan, 1969); High Energy Electromagnetic Processes in Condensed Media (Wiley Interscience, New York, 1972).

[3] G. M. Garibian and Yan Shi, Rentgenovskoe Perekhodnoe Izluchenie (Publishing House of the Academy of Sciences of Armenia, Yerevan, 1983), in Russian.

[4] N. A. Korkhmazian, L. A. Gevorgian, and M. P. Petrosian, Zh. Tekh. Fiz. 47, 1583 (1977).
[5] J. Rosenzweig, G. Travish, and A. Tremaine, Nucl. Instrum. Methods Phys. Res., Sect. A 365, 255 (1995).

[6] E.D. Gazazian, K. A. Ispirian, R. K. Ispirian, and M.I. Ivanian, Pis'ma Zh. Eksp. Teor. Fiz. 70, 664 (1999); Nucl. Instrum. Methods Phys. Res., Sect. B 173, 160 (2001).

[7] A. I. Alikhanian, K. A. Ispirian, and A. G. Oganesian, Zh. Eksp. Teor. Fiz. 56, 1796 (1969).

[8] L. Wartski, J. Marcou, and S. Roland, IEEE Trans. Nucl. Sci. 20, 544 (1973); Proceedings of the International Symposium on Transition Radiation of High Energy Particles, Yerevan, Armenia, 1977, p. 561.

[9] Y. Liu, X. Wang, D. Cline, M. Babzien, J. Fang, J. Gallardo, K. Kusche, I. Pogorelsky, J. Skaritka, and A. van Steenbergen, Phys. Rev. Lett. 80, 4418 (1998).

[10] A. Tremaine, J. Rosenzweig, S. Anderson, P. Frigola, M. Hogan, A. Murokh, C. Pellegrini, D. Nguyen, and R. Sheffield, Phys. Rev. Lett. 81, 5816 (1998).

[11] A.I. Alikhanian, F. R. Arutyunian, K. A. Ispirian, and M.L. Ter-Mikaelian, Zh. Eksp. Teor. Fiz. 42, 2002 (1961).

[12] P. Emma et al., in Proceedings of the 31st International Free Electron Laser Conference (FEL 09), Liverpool, UK (STFC Daresbury Laboratory, Warrington, 2009), p. 397; Nat. Photonics 4, 641 (2010); LCLS, Design Study Report No. SLAC-R-521, 1998.

[13] B. D. Patterson et al., New J. Phys. 12, 035012 (2010).

[14] A.H. Lumpkin, W. M. Fawley, and D.W. Rule, in Proceedings of FEL2004 Conference (Comitato Conferenze Elettra, Trieste, Italy, 2004), p. 515.

[15] A. H. Lumpkin, J. B. Hastings, and D. W. Rule, Report No. SLAC-PUB-12451, 2005.

[16] R. Fiorito, Tenth Workshop on Advanced Accelerator Concepts, CP647, edited by C.E. Clayton and P. Muggli (American Institute of Physics, New York, 2002).

[17] J.D. Jackson, Classical Electrodynamics (John Wiley \& Sons, Inc., New York, 1998), 3rd ed., Chap. 15.

[18] X. Artru, G. B.Yodh, and G. Menessier, Phys. Rev. D 12, 1289 (1975).

[19] Y. Shibata et al., Phys. Rev. E 52, 6787 (1995).

[20] A. G. Adamo, K. MacDonald, Y. Fu, C-M. Wang, D. Tsai, F. García de Abajo, and N. Zheludev, Phys. Rev. Lett. 103, 113901 (2009).

[21] A. A. Tishchenko, A.P. Potilitsin, and M.N. Strikhanov, Phys. Rev. E 70, 066501 (2004).

[22] A.P. Potilitsin, M.I. Ryazanov, M.N. Strikhanov, and A. A. Tishchenko, Diffraction Radiation from Relativistic Particles (Springer-Verlag, Berlin, Heidelberg, 2010).

[23] E. G. Bessonov et al., in Proceedings of the Conference CHANNELING 2010 [Nuovo Cimento (to be published)]; JINST 12, PO510 (2010); arXiv:1003.3747; arXiv: 1009.3724.

[24] Y. Li, W. Decking, B. Faatz, and J. Pflueger, Phys. Rev. ST Accel. Beams 13, 080705 (2010).

[25] G. Geloni, V. Kocharyan, and E. Saldin, arXiv:1106.1776.

[26] M. J. Moran, Phys. Rev. Lett. 69, 2523 (1992). 